Highlighting the Effect of COVID-19 Pandemic on Certain Medically Ill

\title{
Patients: A Scoped Review
}

\author{
Ahmed M. Abbas ${ }^{1,2 *}$, Alaa Rashad Ali ${ }^{2,3}$, Shimaa H. Elsamman ${ }^{2,3}$, Mark Mohsen Kamel ${ }^{2,3}$, Heba Allah Nashaat ${ }^{2,3}$ \\ ${ }^{1}$ Department of Obstetrics \& Gynecology, Faculty of Medicine, Assiut University, Egypt \\ ${ }^{2}$ COvid-19 Research of Assiut UNiversity Association (CORAUNA) group, Egypt \\ ${ }^{3}$ House-officer, Faculty of Medicine, Assiut University, Egypt
}

*Corresponding author: Ahmed M. Abbas, Department of Obstetrics and Gynecology, Assiut University, Women Health Hospital, 71511, Assiut Egypt. Cellular: +20 10033851833; Tel: +20 88 2414616; Fax: +20 88 9202503; E-mail: bmr90@hotmail.com

Received: July 04, 2020

Published: July 30, 2020

\section{Introduction}

Coronavirus disease-2019 (COVID-19) is an infectious disease caused by the newly discovered Coronavirus (SARSCoV2), firstly reported in Yuhan, China in December 2019 [1]. As COVID-19 persists, many countries are adopting restrictive measures to prevent human to human transmission and decrease the virus spread. These measures proven effectiveness in some countries. In this focused review, we will highlight the effect of COVID-19 pandemic on certain groups of medically ill patients.

\section{Organ Transplant Recipients and Donors}

Organ transplant recipients are more susceptible to catch COVID-19 infection rather than other healthy persons. Additionally, they are at risk of more severe disease and symptoms, rapid deterioration, and prolonged viral shedding. All of this attributed to the long term use of immunosuppressant drugs. The risk of superimposed another viral, bacterial, or fungal infection is also high [1].

Prevention and control of COVID-19 disease among this group is a problem for a transplant team. Recipients, donors, and doctors are all at risk. The risks of getting infection include asymptomatic recipients or donor perioperative, false-negative RT-PCR results, recipients' bad immune conditions postoperatively. A management plan for that group includes donors and recipients screening plus donors, recipients, and perioperative management [3]

For donor and recipient screening

- $\quad$ NO contact to suspected or confirmed COVID-19 patients and no history of residence or travel to endemic areas for them and persons in close contact over the past 30 days

- $\quad$ No fever over the past 30 days

- No signs of pneumonia in CT scan

- $\quad$ Negative RT-PCR

- Transfusion blood volume needed present in the blood bank

- For donor management

- Transferred to the respiratory intensive care unit for maintenance

- Accompanied by only one relative
- Donor maintenance personnel are screened

- For recipient management

- Recipients must arrive by private transportation

- Accompanied by only one relative

- For perioperative management

- Implementation of health management for related medical personnel.

- Implementation of an independent operating room and postoperative ward to prevent cross-infection among patients

- Implementation of a professional responsibility system and multidisciplinary treatment

The treatment strategy for immunosuppressed organ transplant recipients infected with COVID-19 was previously reported as case reports [4-6].This depends on the short term stoppage of immunosuppressive drugs to reacquire body immunity to eradicate the virus. Additionally, low dose IV methylprednisolone, prophylactic antibiotic, and antiviral drugs were given. The benefit of steroids is the prevention of acute graft rejection and Addison crisis. The anti-inflammatory effects of steroids also decrease lung exudation and relieve the symptoms. IV steroids should not be used in high doses or for long-duration to avoid inhibition of immunity and side effects of steroids itself.

\section{Osteoporotic Patients}

Prolonged home stay due to quarantine may be associated with side effects that harm people health; one of them is its effect on osteoporosis patients [7].

Osteoporosis is a skeletal disease in which there is disturbance of bone mineralization, low bone mass and bone mineral density, decreased bone strength, and increased risk of fractures [8]. With prolonged home stay in quarantine which may lasts for weeks or even months, there is decrease in mechanical loading and exposure to ultraviolet rays which play a major role in disease progression in osteoporotic patients [7]. Mechanical loading improves bone mass and strength, promotes bone formation through stimulation of osteoblasts differentiation, bone mineralization and decrease osteoclasts activity [9]. Additionally, it regulates hormones in the body such as estrogen, testosterone, parathyroid hormone and glucocorticoids which 
play an important role in bone metabolism and remodeling. Mechanical loading stimulates estrogen production in postmenopausal women which partially mimic hormonal replacement therapy for osteoprosis and Testosterone which reduce bone loss in elderly men [10].

Decrease physical activity due to quarantine leads to increase body weight and obesity which has negative effect on skeletal system $[11,12]$. Insufficient sunlight exposure especially to ultraviolet rays leads to decrease in circulating vitamin D levels (25-hydroxy cholecalciferol) as sunlight exposure mediates the conversion of pre-cholecalciferol (pre vitamin D3) into cholecalciferol (vitamin D3) which then converted into $25(\mathrm{OH})$ D by liver enzymes. Physical activities that can be done without leaving home help to strengthen muscle using safe, simple, and easy exercises such as walking, dancing, using the stair for climbing or steps exercise cardio training and household working [13]. At least 150 minutes of moderate physical activity or 75 minutes of vigorous physical activity per week or combination of them, avoid long-term sitting down, balanced diet containing calcium, protein and vitamin D [14].

Exercise plays a vital role in maintaining a healthy immune system.15 Walking is considered the best form of exercise which doesn't require a gym membership or additional equipment. Brisk walking was selected by researchers when they were testing the relationship between exercise and immune function. Brisk walking is defined as walking at a pace of 2.5 to 4.0 miles per hour. It was found that brisk walking for 3045 minutes per day or, 5 days per week, reduces the respiratory symptoms. The quality of physical exercise a person does influence the immune system. It is affected by the extent and duration reflecting the degree of physiological stress imposed by the workload [15]. Acute bouts of prolonged strenuous exercise cause a temporary depression of various aspects of immune function that last for up to $24 \mathrm{~h}$ after exercise [16].

Although studies are not available for COVID-19, we know that other viruses are much less likely to affect humans who exercise regularly [13]. Intense exercise should be avoided when infected with COVID-19 or other systemic viruses [17]. Sedentary behavior and decreased physical activity can have negative effects well-on the health, being and quality of life of individuals.

For optimal health, it is also important to remember to eat healthily and stay hydrated. Ensure plenty of fruits and vegetables, and limit the intake of salt, sugar and fat. Prefer whole grains rather than refined foods [14]. Psychological stress has a well-known and marked influence on immunity and infection resistance. Athletes may experience psychological stress related to competition, injury, team selection, travel, sleep disruption and jetlag; in addition, .They are more liable to psychological stress in their personal life related to financial hardship and bereavement. Additionally, athletes experience poor sleep patterns compared with non-athletes.

A disrupted night's sleep primes the immune system and ameliorates the immune surveillance by stimulating total lymphocytes, cytotoxic $\mathrm{T}$ cells and natural killer cells to leave the blood and migrate to potential sites of infection during the early recovery period after exercise when compared with normal sleep pattern [18].

In conclusion, physical exercise induces an immune response according to the duration and intensity of exercise. Thus, moderate physical exercise could have an immunoprotective effect and maintain healthy bones especially in the era of COVID-19 pandemic especially for patients with osteoporosis.

\section{Obsessive-Compulsive Disorder Patients}

Pandemic is not considered just a biological or medical event. It could have a psychosocial association, especially with $\mathrm{Ob}$ sessive-Compulsive Disorder (OCD) patients. OCD is mainly manifested by obsessions, which are unwanted thoughts and (compulsions), which are rituals or actions performed repeatedly [19]. With COVID-19 pandemic, obsessions may include fear of contamination, fear of harming self or others, and fear of illness. Compulsions may include excessive washing or bathing [20]. Therefore, COVID-19 can aggravate obsessive and compulsive ideas.

It has been proven that OCD patients, who respond well to pharmacotherapy and psychotherapy, tend to relapse in case of stress due to COVID-19 infection. The increase in symptoms might not be immediate but may take days to months to fully manifest [20]. One of the methods used to prevent the spread of COVID-19 is hand washing. With OCD patients, it is considered trouble as it can be difficult to determine which behaviors are reasonable and which reflect excess fear.

In some developing countries, obsessive-compulsive symptoms are considered to be culture-bound and religion-related, so it is difficult to show people what is "excessive" in this case where everyone fears to follow the preventive measures [21]. Additionally, social media may represent a source of stress to OCD patients through new news about COVID-19 spread.

To contain the pandemic, non-emergent medical services have been suspended in many places. This can further lead to a lack of follow-ups of OCD patients or discontinuation of psychotherapy. Increased stress caused by COVID-19 on OCD patients leads to obsessive use of medications, which may include OCD medications or antiviral drugs [22].

Uncontrolled OCD can lead to chronic stress, insomnia, and a high risk of suicide. Moreover, it can run into a vicious loop, which leads to a chronic sense of vulnerability to the infection, therefore, increase thoughts of hand washing and contamination. These OCD thoughts can be diminished through patient reassurance, following logical advice for health behaviors such as decrease compulsive cleaning rituals, decrease the number of new news views about the virus, decrease travel frequency during the pandemic, cognitive psychotherapy, and medical treatment by suitable drugs.

\section{Cancer patients}

Patient with cancer or those receiving chemotherapy are at higher risk of complications among COVID-19 victims, as it decreases its ability to fight the virus by lowering their immunity. The mechanism by which it works to allow the virus to beat the immune system is firstly all chemotherapy suppresses the bone marrow leading to decrease the white blood cell effect on this system. Secondly, the effect of some cancers on immune system by themselves as leukemia or lymphoma [23]. Regarding the risk of COVID-19 for patients with cancers, some cohort studies found a strong relation between the cancer and the virus. Some studies proved the ability of catching the virus among cancer patients or previously recovered ones who still take immunosuppressive drugs is higher than normal ones. It turns to that their immunity is lowered which make them more likely to catch the disease than others who exposed to the infection source [24]. Patients with cancer who underwent recent therapy or surgery had a higher risk of severe events 
than did those not receiving treatment. The authors concluded that patients with cancer might have a higher risk of COVID-19, and poorer outcomes, than people without cancer. As a consequence, they recommended considering an intentional postponing of adjuvant chemotherapy or elective surgery for people with stable cancers in endemic areas [24].

Additionally, patients using anti-PD-1/PD-L1 or anti-CTLA-4 immune checkpoint inhibitors (ICI) to treat solid tumors such as melanoma, lung cancer, renal carcinoma, urothelial cancers and head and neck carcinoma constitute a growing oncological population. Their susceptibility to bacterial or viral infections has not been investigated yet. Considering that immunotherapy with ICI is able to return the cellular immunocompetence back to its nature [25].

Radiotherapy is a "life-saving" therapeutic modality that should be guaranteed to all cancer patients in which are indicated [26]. There should be solutions for regional and hospital management to ensure the full performance of radiotherapy facilities, even in emergency conditions. A widespread infection of the working staff in a radiotherapy section would result in partial closure of the activities. Failure to identify the suspected or infected patients with COVID-19 would increase the risk of spreading to operators and patients who undergoing treatment [26].

Some precautions were proposed before using radiotherapy facility:

- Verifying the probable contacts with COVID-19 positive patients and prevent those with suspected symptoms from access to the radiotherapy center.

- $\quad$ Providing hand disinfection at the entrance of the radiotherapy center.

- Wearing surgical masks, as recommended for all health professionals and patients according to the WHO indications, and in particular in the following cases: if the physician has respiratory symptoms, to protect others or if the physician is in close contact with a person who has respiratory symptoms, to protect himself.

- Using a sterile disposable gown, masks, clogs, and overshoes when treating patients with suspect COVID-19 positivity, if they indicated to continue the courses of radiotherapy [27].

The use of chemotherapy combined with radiotherapy could increase the risk of morbidity and mortality from concomitant COVID-19 infection. Justification of treatment requires a careful estimation of risk benefit ratios. Patients who may only gain a long-term survival may be advised to avoid radiotherapy during the pandemic. Treatments which reduce loco-regional recurrence, but do not improve survival rates should be avoided during the COVID-19 pandemic.

To sum up, people who suffer from cancer or take immunosuppressive drugs and chemotherapy would be more likely to catch the COVID-19 more than others with a high liability of serious complications.

\section{Conflict of Interest}

The authors state that there are no conflicts of interest.

\section{References}

1. Sohrabi C, Alsafi Z, O’Neill N, Khan M, Kerwan A, Al-Jabir A, et al. World Health Organization declares global emergency: A review of the 2019 novel coronavirus (COVID-19). Int J Surgery. 2020.

2. Fishman JA, Grossi PA. Novel Coronavirus-19 (COVID-19) in the Immunocompromised Transplant Recipient: Flattening the curve. Am J Transplant. 2020.

3. Zhang BH, Yan LN, Yang JY. Organ transplantation management in the midst of the COVID-19 outbreak: a synopsis. Hepatobiliary Surgery and Nutrition. 2020;9(2):250-2.

4. Zhu L, Xu X, Ma K, Yang J, Guan H, Chen S, et al. Successful recovery of COVID-19 pneumonia in a renal transplant recipient with long-term immunosuppression. Am J Transplant. 2020.

5. Huang J, Lin H, Wu Y, Fang Y, Kumar R, Chen G, et al. COVID-19 in post-transplantation patients-report of two cases. Am J Transplant. 2020.

6. Chen S, Yin Q, Shi H, Du D, Chang S, Ni L, et al. A familial cluster, including a kidney transplant recipient, of Coronavirus Disease 2019 (COVID-19) in Wuhan, China. American Journal of Transplantation. 2020.

7. Lippi G, Henry BM, Bovo C, Sanchis-Gomar F. Health risks and potential remedies during prolonged lockdowns for coronavirus disease 2019 (COVID-19). Diagnosis. 2020;7;1. (ahead-of-print).

8. NIH C. Osteoporosis prevention, diagnosis, and therapy. NIH consensus statement. 2000;17:1-45.

9. Zaidi M. Skeletal remodeling in health and disease. Nature Med. 2007;13(7):791-801.

10. Bentz AT, Schneider CM, Westerlind KC. The relationship between physical activity and 2-hydroxyestrone, 16 $\alpha$-hydroxyestrone, and the 2/16 ratio in premenopausal women (United States). Cancer Causes Control. 2005;16(4):455-61.

11. Abbas AM, Fathy SK, Fawzy AT, Salem AS, Shawky MS. The mutual effects of COVID-19 and obesity. Obesity Med. 2020:100250.

12. Mediouni M, Madiouni R, Kaczor-Urbanowicz KE. COVID-19: How the Quarantine could lead to the Depreobesity. Obesity Med. 2020:100255.

13. Rahmati-Ahmadabad S, Hosseini F. Exercise against SARSCoV-2 (COVID-19): Does workout intensity matter? (A mini review of some indirect evidence related to obesity). Obesity Med. 2020:100245.

14. Abbas AM, Kamel MM. Dietary habits in adults during quarantine in the context of COVID-19 pandemic. Obesity Med. 2020:100254.

15. Nieman DC, Wentz LM. The Compelling Link between Physical Activity and the Body's Defense System. J Sport Health Sci. 2019;8(3):201-17.

16. Gleeson M. Effects of exercise on immune function. Sports Sci. Exch. 2015;28:1-6.

17. 17. Zhu W. Should, and how can, exercise be done during a coronavirus outbreak? An interview with Dr. Jeffrey A. Woods. J Sport Health Sci. 2020;9(2):105.

18. Walsh NP. Recommendations to maintain immune health in athletes. Eur J Sport Sci. 2018;18(6):820-31.

19. Davis ML, McIngvale E, Schneider SC, Goodman WK, Storch EA. Obsessive-Compulsive and Related Disorders. InDiagnostic Interviewing. 2019;155-177.

20. Banerjee D. The other side of COVID-19: Impact on Obsessive Compulsive Disorder (OCD) and Hoarding. Psychiatry Research. 2020.

21. Cordell E, Holaway R. Obsessive-Compulsive and Related Disorders. InHandbook of Cultural Factors in Behavioral Health. 2020;293-307.

22. Duan L, Zhu G. Psychological interventions for people affected by the COVID-19 epidemic. Lancet Psychiatry. 2020;7(4):300-2.

23. Tian S, Hu W, Niu L, Liu H, Xu H, Xiao SY. Pulmonary pathology of early phase 2019 novel coronavirus (COVID-19) pneumonia in two patients with lung cancer. J Thoracic Oncol. 2020. 
24. Wang H, Zhang L. Risk of COVID-19 for patients with cancer. Lancet Oncol. 2020;21(4):e181.

25. Bersanelli M, Buti S. From targeting the tumor to targeting the immune system: Transversal challenges in oncology with the inhibition of the PD-1/PD-L1 axis. World J Clin Oncol. 2017;8(1):37.

26. Mukherjee RK, Back MF, Lu JJ, Shakespeare TP, Wynne CJ.
Hiding in the Bunker: Challenges for a radiation oncology department operating in the Severe Acute Respiratory Syndrome outbreak. Australasian Radiol. 2003;47(2):143-5.

27. Filippi AR, Russi E, Magrini SM, Corvò R. COVID-19 outbreak in northern Italy: first practical indications for radiotherapy departments. International Journal of Radiation Oncology* Biology* Physics. 2020. 